\title{
Responsive complex surfaces manufacturing using origami
}

\author{
Mara Capone ${ }^{1}$,Emanuela Lanzara ${ }^{2}$, Laura Marsillo ${ }^{3}$, \\ Carlos Alejandro Nome Silva ${ }^{4}$ \\ ${ }_{1,2,3}$ University of Naples Federico II ${ }^{4}$ UFPB Universidade Federal da Paraiba \\ ${ }^{1,2}\left\{\right.$ mara.capone|emanuela.lanzara\}@unina.it ${ }^{3}$ laura.mars92@gmail.com \\ ${ }^{4}$ carlosnome@ct.ufpb.br
}

Contemporary architecture is considered a dynamic system, capable of adapting to different needs, from environmental to functional ones. The term 'Adaptable Architecture' describes an architecture from which specific components can be changed in relation to external stimuli. This change could be executed by the building system itself, transformed manually or it could be any other ability to be transformed by external forces (Leliveld et al.2017). Adaptability concept is therefore linked to motion and to recent advances in kinetic architecture. In our research we are studying the rules that we can use to design a kinetic architecture using origami. Parametric design allows us to digitally simulate the movement of origami structures, we are testing algorithmic modeling to generate doubly curvature surfaces starting from a designed surface and not from the process. Our main goal is to study the relationship between geometry, motion and shape. We are interested, in particular, in complex surface manufacture using origami technique to design a kinetic and reactive ceiling.

Keywords: Origami, complex surface manufacture, responsive architecture, Applied Geometry

\section{INTRODUCTION}

Contemporary architecture is considered a dynamic system, capable of adapting to different needs, from environmental to functional ones. The term "adaptable architecture" describes an architecture from which specific components can be changed in relation to external stimuli, for example the users or environment. This change could be executed by the building system itself, transformed manually or it could be any other ability to be transformed by external forces (Leliveld et al.2017). Adaptability concept is therefore linked to motion and to recent advances in kinetic architecture.

Applied geometry and computational approach are basis in order to define tools to test different configurations that a shape can assume by dynamically changing its appearance.

In our research we are studying the rules that we can use to design a kinetic architecture using origami. We are going to test the potential of procedural modeling in this field.

Parametric design allows us to digitally simulate 
the movement of origami structures, we are testing algorithmic modeling to generate doubly curvature surfaces starting from a designed surface and not from the process. Our main goal is to study the relationship between geometry, motion and shape. We are interested, in particular, in complex surface manufacture using origami technique to design a kinetic and reactive ceiling.

Fold is great both for static and kinetic reasons. The bending of a surface produces a stiffening in direction of fold itself that is based "on increasing the bearing capacity of a structural element "(Rodonò 2016). Using the folding mechanism you can design a dynamic shape that moves and transforms in relation to different pattern you used to discretize the surface.

Digital fabrication technologies are evolving and becoming more and more widespread. Knowledge of fabrication methods available and parametric design tools, based on geometry, are changing the designer way of thinkng.

The geometry that governs folding surfaces derives from Oriental art world of origami, whose structuring element is the fold (Rodonò' 2016). The term comes from Japanese origami: ORI = fold and KAMI = paper, the art of paper folding. The purpose of this art was to get forms using only folds without glue and cuts.

We can distinguish the origami in two categories (fig. 1): 1) Traditional origami, where the use of the fold is used to accomplish a very specific form, 2) Geometric Origami, which investigates the properties that the fold is able to determine the surface and spatial configurations (Hassan 2004). The main element is the fold, which becomes a hinge and allows movement and a stiffening along the direction of development, and tessellation is the typical element of the building (Casale 2012). We can distinguish three structural families of folds, which determine three types of configurations (fig.2): 1. Chaotic: the plan is subjected to a very dense tessellation and messy. The plan can take endless configurations. 2 . Form: the surface is subjected to a tessellation composed of polygons choices for shape and disposition, for a specific configuration. 3. Structural: determined by dividing the plane into pieces equal to each other and lets assume in space many possible configurations.

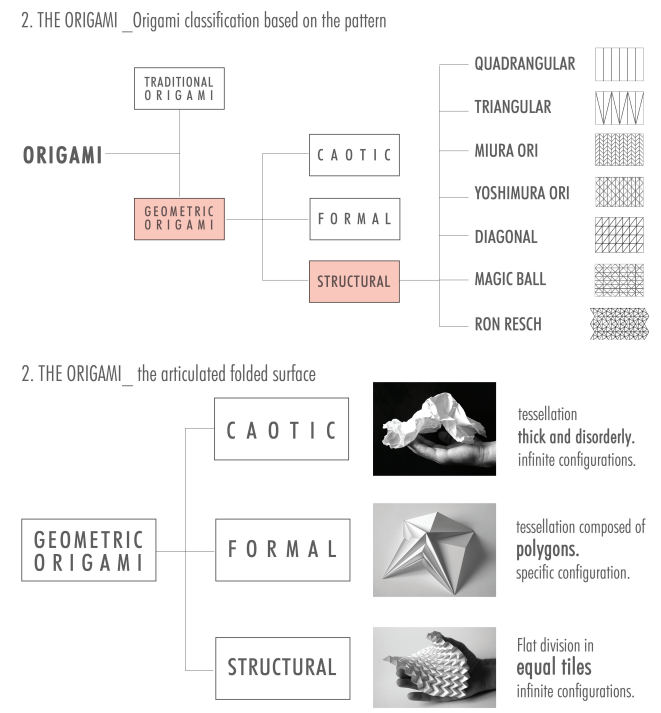

Our goal is to investigate the surface and spatial configurations that we are able to generate using folding technique. From a methodological point of view we have analyzed historical applications, contemporary references and a lot of parametric tools that are defined in recent years to test new processes to generate a kinetic and responsive application. We are going to manufacture a ceiling prototype that is able to move according to different acoustic requirements. We used parametric tools to generate the shape and to simulate the movement. Our aim is to create a ceiling, using origami technique, that is able to move using less mechanisms as possible.

\section{STATE OF THE ART} Historical background and related work

In the 20th century, many architects began to be interested in complex shapes design based on origami
Figure 1

Origami classification

Figure 2

Geometric Origami classification 
knowledge. In the German Bauhaus school, Joseph Albers encouraged his students to study and experiment with new shapes using different materials (MAGRONE 2015). During the 1950s the art of origami became famous around the world thanks to the artist Aikira Yashiwazawa. In the 1960s and 1970s, Ron Resch, a visionary mathematician and designer, was one of the first to explore the potential of 3D Architectural mosaic structures. His Triangle Fold is the basic geometry for many foldable surfaces prototypes with a range of dynamic systems usable in different fields. During 1970 Symposium, that took place at Tokyo University Institute of Space and Aeronautical Science, the astrophysicist Koryo Miura showed his design for a solar panel for space satellites made of quadrangular elements equal and foldable that was called "Miura fold pattern" (CASALE 2012). In our research we are experimenting with Miura Ori, Ron Resch and Magic ball patterns. We have analyzed different adaptable projects according to different ways to generate movement systems. We have determined different approaches and we have therefore grouped the case studies into two different groups: 1) systems composed by elements that can move independently of each other, in this case the design shape is dicretized using these elements; 2) continuous systems that can be generated folding a flat panel, in this case we can obtain different shapes applying forces. We are interested in the second group and we are going to show a case study consisting in a responsive ceiling that reacts to different environmental impulses (for acoustic applications) using Arduino technology. Main features of our project are: transformability, transportability, use of the least number of engines for movement, construction with use of low-cost materials.

\section{Theoretical framework}

In our research using origami technique we can transform a flexible but non-deformable panel into an "origami panel" that is flexible and deformable. From a theoretical point of view using a flexible panel we can manufacture a developable surface (zero Gaus- sian curvature) but we can't manufacture a complex shape (negative or positive Gaussian curvature), on the contrary we can manufacture all kind of surface using a deformable panel. In this case, we have to determinate the degree of deformability according to the designed shape Geometric genesis of surfaces and knowledge of their properties are basis for solving many problems, both constructive and measurement. Our research in the field of non-developable surfaces fabrication move from "bending" (origami) to kerf-bending (kirigami). As Monge teaches, surfaces can be distinguished, in comparison to the curvature, in three large categories. The first category includes those surfaces that in their points do not have any kind of curvature and they are flat surfaces. The second category concerns those that in each of their points have only one curvature and are, fundamentally, all the surfaces that can be developed. The third category of surfaces is composed of all the others that are called double-curved surfaces. The curvature of a curve in $P$ is $k$, where $k=1 \backslash r$ and $r$ is the radius of the osculating circle of the curve, we can define the main sections of a surface the sections of the surface, obtained with planes passing through the normal to the surface in $\mathrm{P}$, with minimum and maximum curvature. Gaussian curvature is the product of the two main curvatures, so it can be positive, negative or equal to zero: it is positive when the osculating circles of the main sections are on the same side of the tangent plane, negative when they are on opposite sides, zero when one of the two main sections is a straight line. The surfaces with zero curvature are specific ruled surfaces, also called developable. As we know each developable surface can be flattened onto a plane without distorsion and, in a limited region, without overlapping so this kind of surfaces can be obtained easily bending or rolling of flat sheets (fig.3). We know how to determinate the develop of a developable surface so there aren't problems to solve. In the case of doubly curvature surfaces manufacturing (negative or positive) using a flat panel we have to make material deformable and we have to define "approximate devel- 
opment" or "planarization" of the non-developable surfaces. In order to manufacture complex surface using origami technique we also have to solve problems related to the way of determining the relationships between 3D surface and the "origami panel", the shape of "origami panel" to obtain the designed surface. This shape depends on degree of deformability, on pattern and its size, the "scale".We have studied the different kinds of geometric origami and we have manufactured physical models to demonstrate and compare their feasibility. After carrying out these series of analysis aimed at defining the parameters that affect the folded surface, we have selected three patterns that we can use to generate surface with positive and negative Gaussian curvature. We have defined parametric model to control the relationship between spatial configuration, which the bent surface can assume, and pattern "scale" in relation to the starting surface.

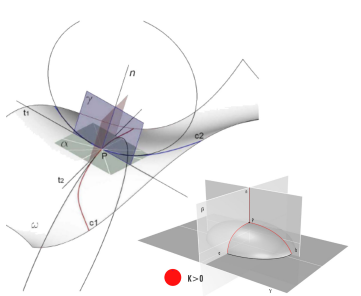

\section{GEOMETRY/CURVATURE RATIO}
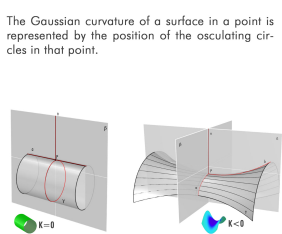

Pattern classification according to the curvature surface

\begin{tabular}{|c|c|c|c|}
\hline PATTERN & $\begin{array}{l}\text { POSITIVE } \\
\text { CURVATURE }\end{array}$ & ( & * NEGATIVE \\
\hline auadrangular & / & plane & / \\
\hline$W$ trangular & / & plane, cono, cylinder, cilindroide & hyperbolic paroboloid \\
\hline$\searrow$ mURA ORI & sphere & plane, cono, cylinder, cilindroide & $\begin{array}{l}\text { hyperbolic paraboloid } \\
\text { hyperboloid hypertolic }\end{array}$ \\
\hline$\bigotimes$ diamono & / & cylindical, barrel voult & / \\
\hline$N_{\text {diggonal }}$ & / & Elliptical quadric cylinder & / \\
\hline$\bowtie \triangle$ MaGIC BALL & sphere, sphenical shell & plane & hyperboloid hypertolic \\
\hline$\triangle$ RON RESCH & spherical shell & borrel voult & hyperbolic paraboloid \\
\hline
\end{tabular}

This is a very important think to consider because the different configurations that we can obtain depends on the subdivision of the starting surface (geometric tasselization). For example, by dividing the initial surface into eight parts, using the Ron Resch pattern, we will have the surface succeed in obtaining a certain configuration, which will be different from the final configuration if the starting surface is divided into thirty-two. We will obtain a "planar origami surface" that is more flexible and more deformable if the initial surface to be folded is more and more divided. Using this "planar origami surface" we can manufacture a doubly curvature surface. It can be deduced that, in addition to the geometrical characteristics of origami patter used, we also have to consider that the pattern "scale" influences the possible spatial configurations that the folded surface can assume.

\section{APPLIED GEOMETRY FOR STRUCTURAL ORIGAMI Workflow}

This is the workflow of our research: 1.Analysis of different origami patterns in relation to geometric features; 2 . Tests: manufacturing a doubly curvature surface starting from "origami panel"; 3. Compare test using different origami patterns (tiles shape); 4 . Tests origami tessellation in relation to tiles scale; 5. Parametric models generation to simulate movement and shape morphing.

Origami tessellations are essentially patterns, folded with origami, that repeat themselves as long as you want to continue folding. The main variables of this system are two: the size of the module and the size of the plan. When a non-deformable module is assigned, tessellating a given surface could be impossible problem to solve. We have done a definition that allows us to evaluate the difference between the given surface and the tessellated surface without pattern deformation. Our main goal is to find an optimized solutions. First we studied the different possible ways to parameterize the patterns that we have used in our research: Magic ball, Miura Ori and Ron Resch.
Figure 3

Gaussian Curvature

Figure 4

Structural Origami classification according to Gaussian curvature 
Figure 5

Ron Resch

tessellation:

parametric tools

Figure 6

Surface tessellation using Ron Resch pattern
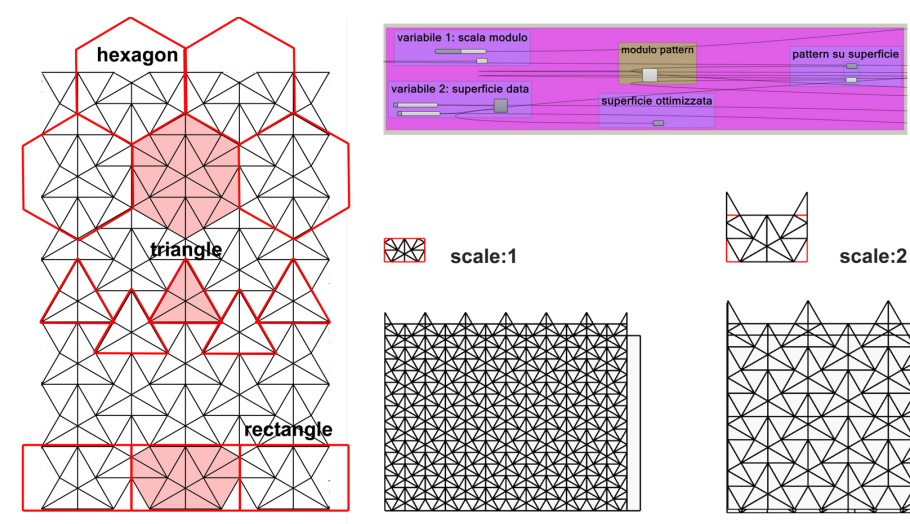

$\$ 4$

scale: 1

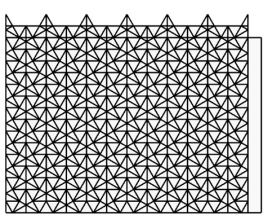

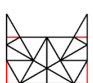

scale:2

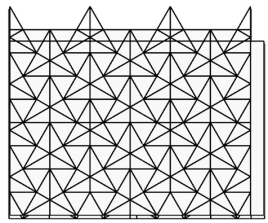

scale:3

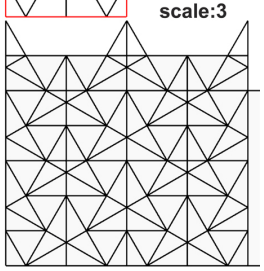

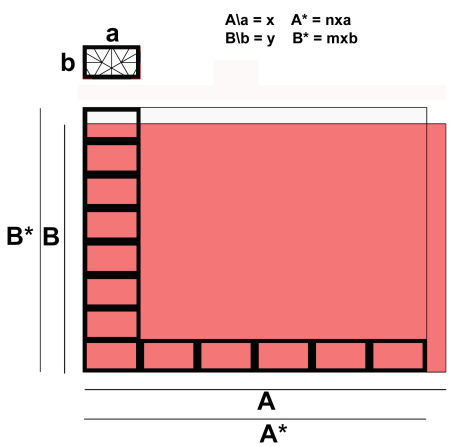
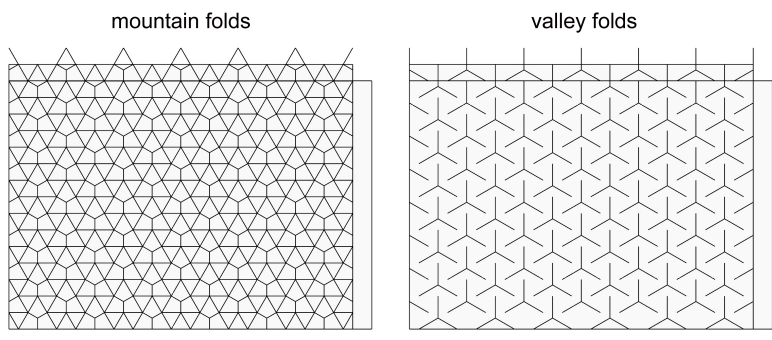

Ron Resch module for tessellation. We have identified three different modules in the geometry of the Ron Resh pattern: the rectangular module, the triangular module and the hexagonal module. In the first case assigned a rectangular surface ( $A$ and $B$ sides) and defined the pattern size ( $a$ and $b$ sides), the number of modules for surface tasselization is: $A: a=x, B: b$ $=y$. The sides of "tessellable" surface will be equal to $A=n \times a$ and $B=m \times b$. If $a$ is not a divisor of $A$ and $b$ is not a divisor of $B$, we have to approximate $x=n$ and $y=m, n$ and $m$ are numeric values of $x$ and $y$ expressed as a whole number without decimal places.
We proceeded considering the hexagonal and triangular modules in a similar way (figs. 5,6).

\section{Relationship between pattern and curva- ture}

The main goal of our research is to define tools able to find optimized solutions to manufacture doubly curvature surfaces using origami. We think that the relationship between pattern size and curvature is the key point to solve this problem. We have done some tests to analyze the relationship between pattern size and curvature using different patterns. From 
a methodological point of view we have used three different "origami panels" with three different tasseletion size.

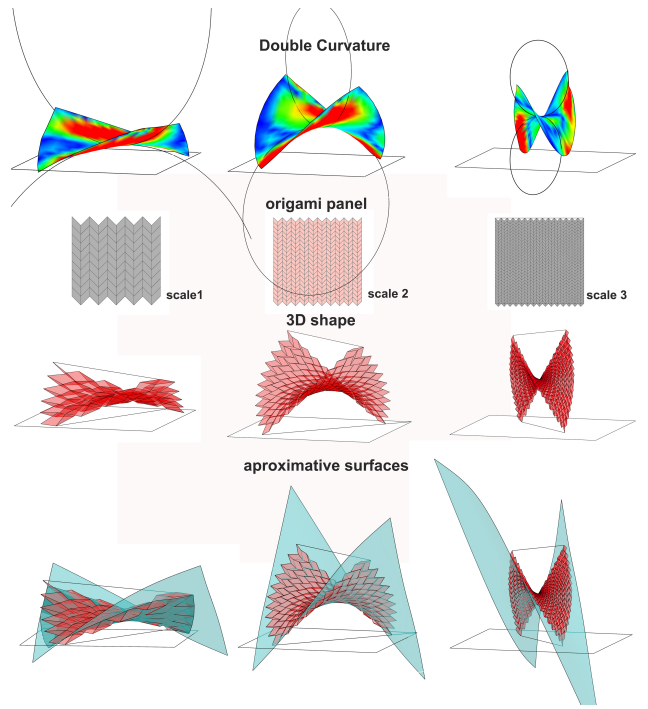

We have moved these panels in order to get a double curvature surface close to an hyperbolic paraboloid.Our goal is to analyze the relationship between pattern size and curvature so we have generated an approximate surface to evaluate the main curvatures. From the comparison between our experiments emerges that using the Miura and the Magic Ball patterns the relationship between curvature and pattern size is direct: reducing pattern size the surface is more "flexible", decreases the radius of osculating circles and consequently increases the main curvatures (fig. 7). Our aim is to find relationship between the pattern size used for flat surface tessellation and the main curvature of 3D shape. Regarding to the Ron Resh pattern this rule is respected only within a given range (fig.8). On the contrary, by reducing the pattern size beyond the defined limit, the surface becomes stricter. By critically analyzing of this result emerges that a thicker tessellation of the surface does not correspond to a greater "flexibility" because the shape moves between two limit configurations: the initial, flat, and the final one, in which it can no longer move because the sides or vertices of the parts of which it is composed touch each other (Casale p.23). Our tests help us to find the relationship between pattern size used for flat surface tessellation and main curvature of 3D shape.

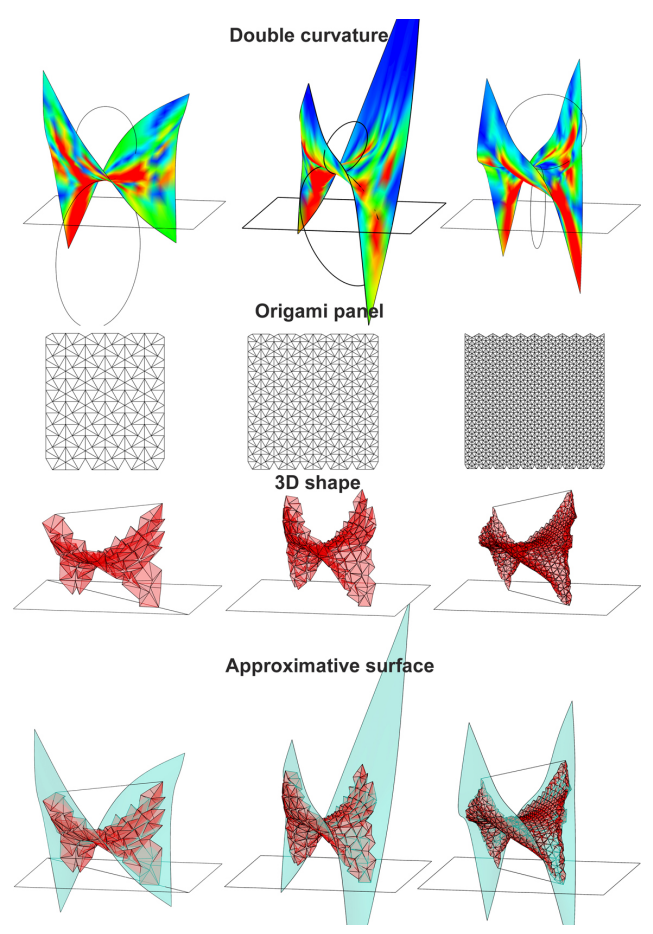

CASE STUDY: DESIGN RESPONSIVE CEILING (DOUBLY CURVATURE SURFACE MANUFACTURING)

\section{Acoustic geometry}

Our aim is to develop a kinematic and versatile system, capable of optimizing the diffusion, concentration or absorption of sound within a multi-functional
Figure 7

Miura Pattern: relationship between pattern scale and curvature

Figure 8

Ron Resh pattern: relationship between pattern scale and curvature 
Figure 9

Acustic geometry environment in response to specific activities for which each area is intended. Therefore, the complexity of the formal-geometrical configuration of such a partition system (vertical and/or horizontal) will depend on the acoustic needs of a specific environment.

The acoustic phenomenon is composed of three main elements: the source, the path and the receiver. The path is the medium through which sound source transmits the sound, and it can be air or a built system. Sound is a mechanical wave that propagates through a medium: the speed of sound varies according to the elasticity and density of the medium, and therefore of the material used (Adams, 2016). When sound meets a surface, part of the energy is absorbed, part is reflected and part is transmitted through the medium: material properties influence all these characteristics. When surface is flat, the angle of reflection is equal to the angle of incidence. If sound waves, even if configurable as rays, encounter a curved surface, the modes of reflection vary according to the curvature of the surfaces that configure the partition system chosen for the internal spatial configuration of a given environment (fig.9). In particular, convex surfaces diffuse sound, while concave surfaces concentrate it. These reflections open up a question: can an acoustically configured kinematic system be responsive or customised? In the first case, the kinematic nature of the system depends on how sound waves propagate: so, sound parameter, according to specific needs, will activate kinematic system that regulates in such a way as to position the panels until a specific curvature is reached to allow the concentration or diffusion of the sound emitted by the source. In the second case, the situation is reversed: curvature of the system surfaces, and therefore the shape, is the starting parameter in relation to which the designer will optimize the concentration or diffusion of the sound according to his needs. Consider the second case for which the system conformation is customized according to specific needs. If, for example, inside the room it will be necessary to set up areas in which to have a sound concentration and an isolation with respect to the external environment, the part of the system located near this area will assume a concave configuration and the panels that make up the pattern will be made using a reflective material. On the contrary, the panels that make up the part of the system facing outwards will be made using an absorbent material (fig. 10). Another fundamental aspect, therefore, is the choice of materials linked to the acoustic purpose. They are divided into sound absorbing and sound insulating materials. The purpose of sound absorbing materials is to transform a part of the sound wave into another form of energy, usually heat, reducing its intensity.

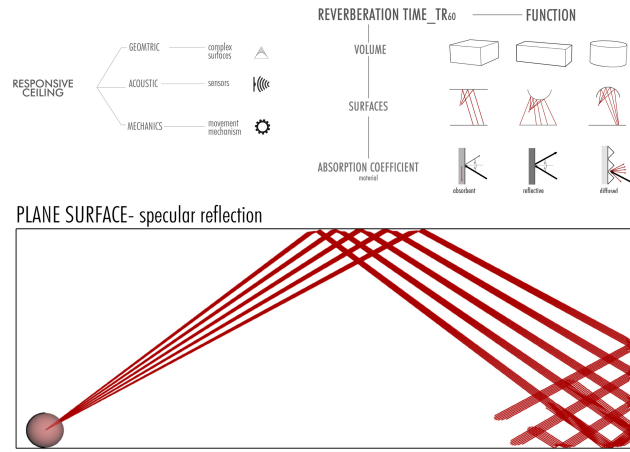

CONVEX SURFACE - diffusion

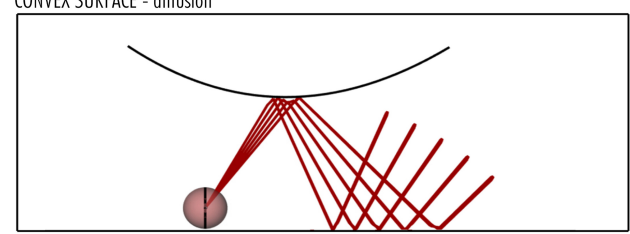

CONCAVE SURFACE- concetration

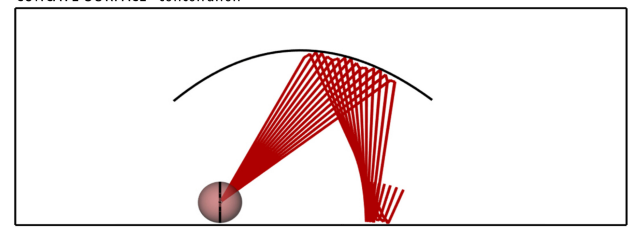




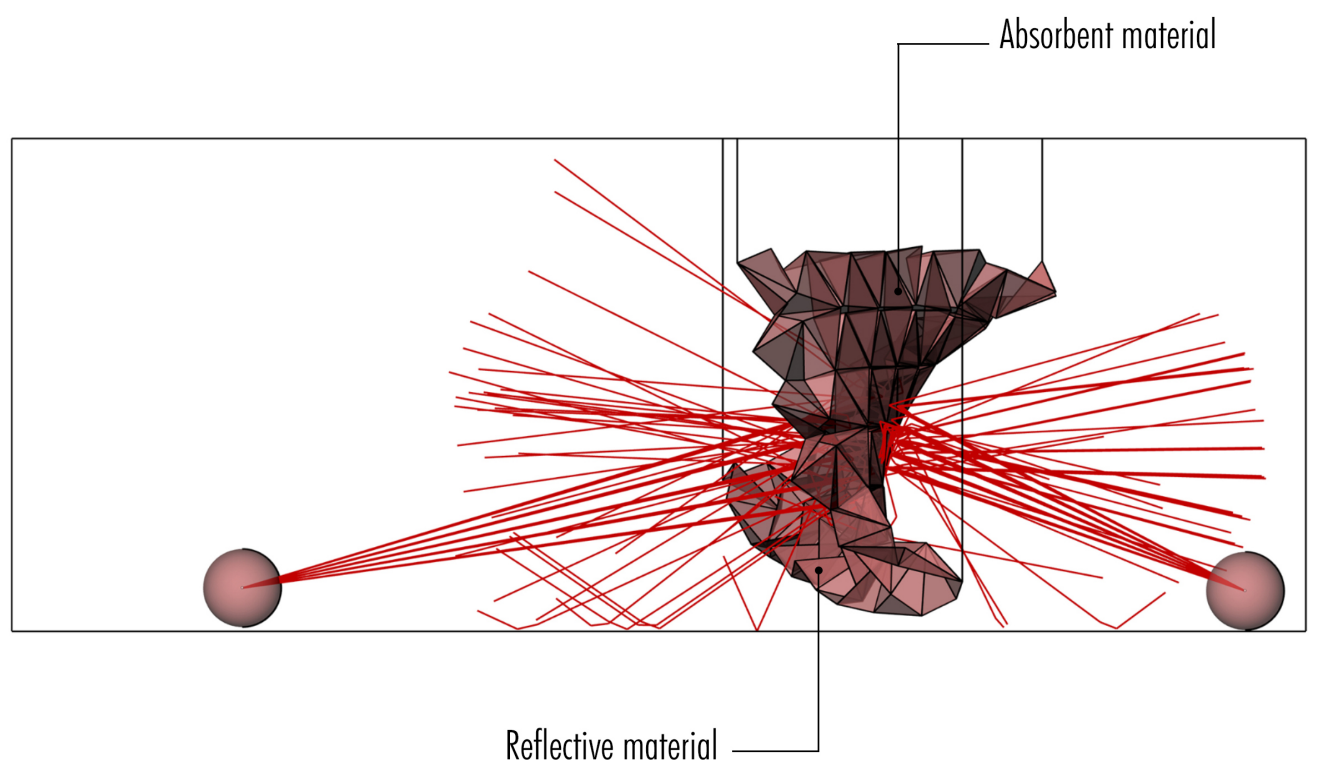

Figure 10

Customised system

Absorbent materials are materials considered porous, which allow the penetration of air and therefore acoustic energy, such as glass wool, stone, natural fibers, bamboo, jute and so on. These sound absorbing materials are used to adjust the noise level in enclosed spaces, to reduce reverberation time, to eliminate echoes, etc. While the sound insulating materials have the characteristic of reflecting the sound and therefore do not dissipate it into the environment. For example, such as rubber, lead, polymeric membranes, wood, etc., the sound is reflected in the room. Therefore, it is possible to make a further classification of materials in relation to digital manufacturing by using CNC machines, 3D printers, etc. This means that the elements that make up the pattern could be made using two different materials, then proceeding to identify the clusters of panels based on the shape and material to optimize the phases of digital manufacturing and assembly of the system. The following paragraph clarifies the distri- bution of the panels in relation to the configurations assumed by the system.

\section{The mechanical system}

One of the main goal of our research is to design a system that is able to fold and unfold in one continuous motion using a simple mechanical system. It consists of a main structure made up of main tracks connected to each other by rigid transverse rods. Assuming that the structure is associated with a Cartesian reference system, we can consider the main tracks oriented in the $x$ direction and the crossbars in the $y$ direction. The main tracks are connected to transverse secondary tracks that run along the direction $\mathrm{x}$ on which in turn runs a system of wheels along the direction $y$ to which the reels are attached. The reels control the movement in the $z$ direction by winding and releasing the connecting cables between the upper frame and the vertices of the origami.This mechanism allows to use the behaviour at the base of the 
Figure 11

Mechanical testing for acoustic ceiling motion origami, allowing to realize the different configurations in space thanks to the movement of certain vertices of the ceiling (fig.11).

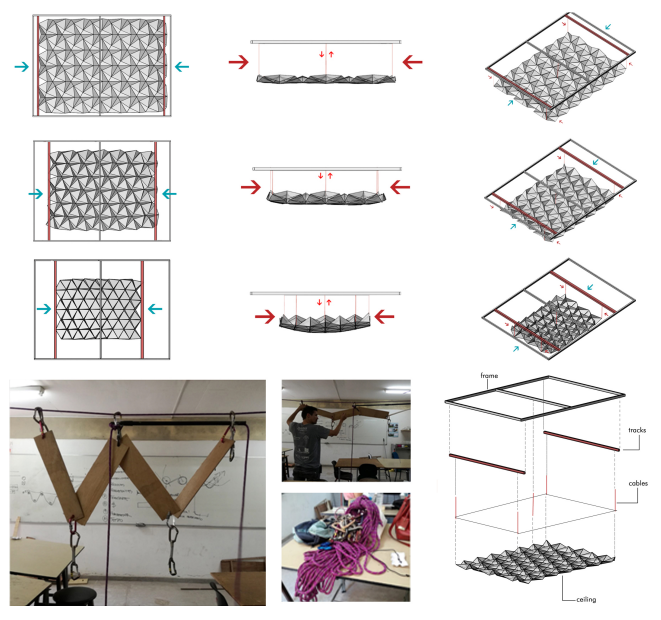

From digital model to manufactured system

The physical system presents some problems caused by the material thickness. It is composed of side $A$ and side $\mathrm{B}$ complementary to each other as a consequence of the kinematic motion allowed by the specific typology of origami pattern. The folds consist of intersection segments between pairs of adjacent faces: according to the different side they behave simultaneously as valley folds or upstream folds. The consecutive dihedrals identified by pairs of adjacent faces will have a concave or convex angle according to the system configuration. The origami - pattern is composed of open concave and convex polyhedra: the open concave polyhedra (side A) are at the same time convex polyhedra (side B) according to the specific design requirements.In open concave polyhedra, shown in red (fig. 12), the thickness of the panels does not allow the final configuration and causes a collision between the consecutive faces.

Therefore, in order to optimize digital fabrication and assembly, the thickness of the panels must be outgoing from the convex portion determined by the kinematic motion induced on the system (Fig.12).

About fabrication and assembly process there are two hypotheses based on the rule described above. The first hypothesis involves the inclusion of an insulating membrane between the rigid panels (Fig.13 solution 1). The visual scripting approach allows to control the size of the phono-absorbent sheath bands by offsetting the edges of the triangular faces on both sides using the same value of the material thickness. Therefore, it is necessary to distance the panels to allow the complete adjacency of the faces that compose the concave polyhedra. This system allows to avoid any collision between the faces during kinematics and according to the final configuration.

The second hypothesis is a digital stereotomy experiment using materials suitable for a milling cutter with 5 or 6 axes. Stereotomic techniques advance according to the development of geometric knowledge and the advancement of digital tools. Stereotomy can be considered as the science that studies the geometric tracing of solids to be obtained with cuts, even if only theoretically, from other solids, the interpenetration between solids and the identification of intersection lines and curves. According to these kinematic experiments the selected material is thicker than paper or cardboard. Therefore, it is necessary to calculate the tapering of adjacent faces to allow specific configurations. According to this hypothesis, the tapering of each panel is parametrically controlled according to the thickness of the chosen material and the concave or convex bending angle between groups of adjacent faces. The faces tapering proceeds from the central area to the edges of the panels and the difference for each panel is directly related to the complexity of the designed spatial configuration. Therefore, the angle tapering depends on two parameters: material thickness and bending angles. This solution could include hinges along all edges of the panels to allow the folding system (Fig. 13 solution 2). The first hypothesis optimises the digital manufacturing process of the panels. In addition, it will be possible to avoid the additional 


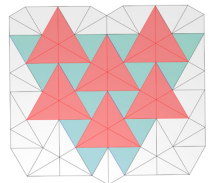

a

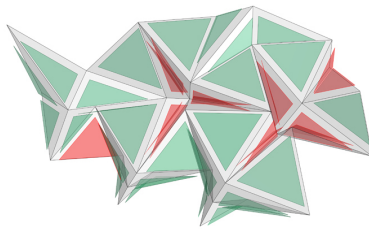

cost of the hinges to allow kinematics.

Anyway, it is possible to consider an intermediate hypothesis involving adjacent tapered panels on an intermediate insulating membrane (Fig.13 solution 3). However, the position of the panels according to the concave or convex areas determines voids in terms of material performance.Therefore, for all hypotheses it will be necessary to insert thin panels of reflective or absorbent material to cover the empty areas or to complete the back of the panels according to the solution that involves the use of hinges along the edges.
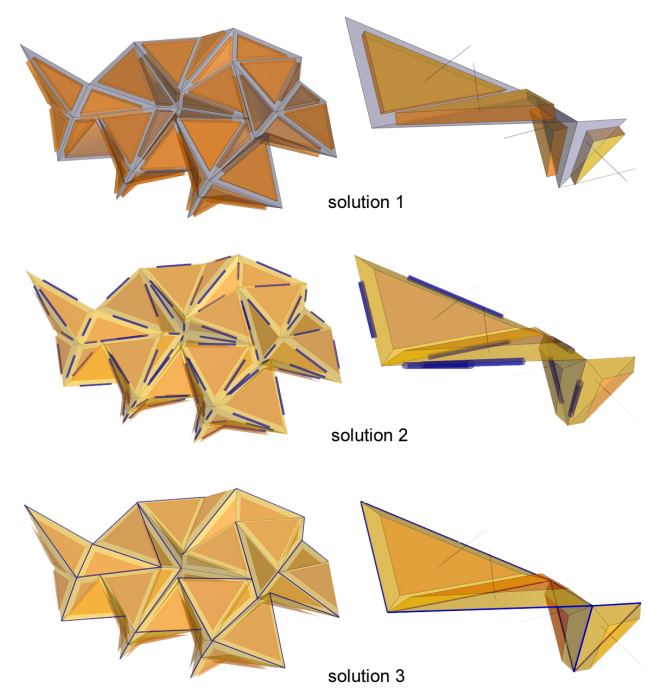

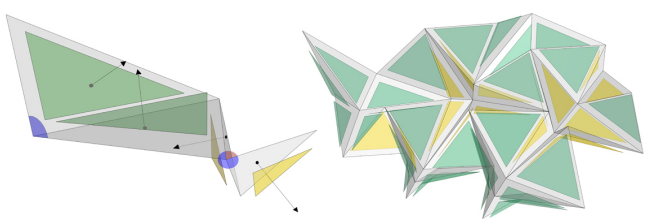

b

\section{CONCLUSIONS AND FUTURE WORKS}

In conclusion, all the hypotheses described above will be investigated to determine which solution provides the best kinematic, economic and aesthetic solution. We are going to define a computational tools based on origami tesselation rules and digital stereotomy principles.

\section{REFERENCES}

Adams, T. 2016, Sound Material. A compendium of Sounds Absorbing Materials for Architecture and Design., Frame Publishers, Amsterdam

Callens, S. J. P. and Zadpoor, A. A. 2018, 'From flat sheets to curved geometries: Origami and kirigami approaches.', Materials Today, 21(3)

Casale, A. and Valenti, G. M. 2013, 'Architettura delle superfici piegate. Le geometrie che muovono gli origami', in Migliari, R., Casale, A., Cinti Luciani, S., Fasolo, M., Trevisan, C. and Valenti, G. M. (eds) 2013, Nuovi quaderni di Applicazioni di Geometria Descrittiva, Aracne Editrice, Roma

Frogheri, D., Estévez, A. T. and Carlos, F. M. 2017 'Resilient Morphologies: from digital to matter', SIGraDi 2017, Concepción, Chile

Gardiner, M. 2018 'Mapping: Parametric Design of Origami Surfaces with Periodic Tessellations', 7th Origami Science Mathematics and Education Conference Oxford, United Kingdom

Lelieveld, C., Voorbij, A.I.M and Poelman, W.A. 2007 'Adaptable architecture', Building Stock Activation, Tokyo

Magrone, P. 2015 'Form and art of closed crease origami.', Conference on Applied Mathematics APLIMAT2015, Slovakia

Rodonò, G. and Sapienza, V. 2016, 'KREO- Kinetic Responsive envelop by origami', TEMA, vol. 2, pp. 4248
Figure 12

a) Bending

problems linked to panel thickness b) Geometric solution according to panel thickness
Figure 13

solution 1: inclusion of an insulating membrane between the rigid panels; solution 2: based on digital stereotomy; solution 3: intermediate hypothesis. 